\title{
THE REPRESENTATION OF D. G. NEAR-RINGS
}

Dedicated to the memory of Hanna Neumann

\author{
J. D. P. MELDRUM
}

Communica:ed by M. F. Newman

(Received 27 June 1972)

In this paper we study the problem of the representation of d.g. near-rings, and in particular the problem of a faithful representation, which is equivalent to the adjoining of an identity. This problem has been considered by Malone [5] and Malone and Heatherly [6] and [7]. They have shown that a finite near-ring with two sided zero can be embedded in the d.g. near-ring generated by the inner automorphisms of a suitable group, and that an identity can always be adjoined to a near-ring with two sided zero. They have also given some special conditions under which a faithful representation of a d.g. near-ring exists.

From another point of view, Fröhlich has studied groups over a d.g. nearring in [3] and [4]. If $(R, S)$ is a d.g. near-ring, where $S$ is the distributive semigroup generating $R$, then he showed that free $(R, S)$ groups exist. We use free $(R, S)$ groups to show that not every d.g. near-ring $(R, S)$ can have a faithful representation on a group, if we insist that $S$ should be a semigroup of distributive elements, i.e. endomorphisms on the group. This is true even in the finite case.

We start by setting the work of Fröhlich on free $(R . S)$ groups in the context of varieties, using methods differing substantially from his. Using these ideas, we construct in each non-abelian variety a d.g. near-ring without a faithful representation. This opens up the problem of determining those d.g. near-rings which do have a faithful representation. It also leaves open the question of whether it is possible to embed a d.g. near-ring $(R, S)$ in a d.g. near-ring with identity, if we do not insist that the elements of $S$ should be distributive in the larger near-ring.

We finish by establishing that for every d.g. near-ring $(R, S)$, there exist 'nearest' d.g. near-rings $(\bar{R}, S),(\underline{R}, S)$ which have faithful representations and such that $(R, S)$ is a homomorphic image of $(\bar{R}, S)$ and $(\underline{R}, S)$ is a homomorphic image of $(R, S)$. For those d.g. near-rings to which an identity can be adjoined, there is a natural way of doing it. If the near-ring is a ring, then it is interesting to note that this method of adjoining the identity is the standard one. 


\section{Definitions and preliminary results}

A near-ring $R$ is a set on which are defined two operations: addition + and multiplication $\cdot$, a symbol which will generally be omitted, such that $(R,+)$ is a group (not necessarily commutative), $(R, \cdot)$ is a semigroup and the left distributive law

$$
x(y+z)=x y+x z \text { for all } x, y, z \in R
$$

is satisfied. The additive identity will be denoted by 0 , and the multiplicative identity, if it exists, by 1 . An element $r \in R$ is called distributive if we have

$$
(x+y) r=x r+y r \text { for all } x, y \in R .
$$

A distributively generated (d.g.) near-ring is a near-ring $R$ such that $(R,+)$ $=G p\{S ; S$ a (multiplicative) semigroup of distributive elements $\}$, i.e. $R$ is generated as an additive group by the set $S$. $S$ need not be the semigroup of all distributive elements. As the set $S$ which is chosen can be important, we denote a d.g. near-ring by $(R, S)$.

A common example of a near-ring is the set of all mappings of a group $G$ (all groups will be written additively) into itself, with point-wise addition, and with multiplication being composition of maps. Distributive elements in this case are the endomorphisms, and these generate a d.g. near-ring, which we will denote by $E(G)$. A pair $G, \theta$ consisting of a group $G$ and a near-ring homomorphism $\theta$ is called an $(R, S)$ group, where $(R, S)$ is a d.g. near-ring, if $\theta$ is a homomorphism from $R$ into $E(G)$ such that $S \theta \subseteq \operatorname{End}(G)$, the semigroup of endomorphisms of $G$. Such a map $\theta$ is called a representation of $(R, S)$ on the group $G$. The map will often be omitted and we will write $g r$ for $g(r \theta)$, where $g \in G, r \in R$, and speak of an $(R, S)$ group $G$. A representation $\theta$ is faithful if $\operatorname{Ker} \theta$, the kernel of $\theta$, is trivial. If $R$ has an identity 1 , the representation $\theta$ will be called unitary, and $G$ will be called a unitary $(R, S)$ group, if $\theta$ maps 1 to the identity map of $G$ onto itself. Every d.g. near-ring $(R, S)$ has an obvious representation, namely the right regular representation on the group $(R,+)$, which is faithful if $R$ has a left identity, i.e. an element $e$ such that $e r=r$ for all $r \in R$.

Let $S$ be a multiplicative semigroup. Then a group $G$ is called an $S$ group if there exists a homomorphism $\theta$ from $S$ into $\operatorname{End}(G)$. We then write $g s$ for $g(s \theta)$, where $g \in G, s \in S$. A homomorphism $\phi$ from an $(R, S)$ group ( $S$ group) $G$ to another $(R, S)$ group ( $S$ group) $H$ is an $(R, S)$ homomorphism ( $S$ homomorphism) if

$$
(g r) \phi=(g \phi) r \text { for all } r \in R,
$$

$((g s) \phi=(g \phi) s$ for all $s \in S)$. Fröhlich has proved $([2], 2.1 .1)$ that $\phi$ is an $(R, S)$ homomorphism if and only if it is an $S$ homomorphism. Near-ring homomorphisms are not sufficient for our purposes. So we define a d.g. near-ring homomorphism as follows. Let $(R, S)$ and $(T, U)$ be two d.g. near-rings. Then a d.g. near- 
ring homomorphism $\theta$ is a near-ring homomorphism from $R$ to $T$ such that $S \theta \subseteq U$.

We will now define the varieties with which we will work. A variety of groups is the class of all groups satisfying a given set of laws or words, e.g. the variety of abelian groups is the class of all groups satisfying the law $[x, y]=-x-y+x+y=0$. See H. Neumann [8]. Varieties of d.g. near-rings can be defined in the same way, but by using laws involving both addition and multiplication. These have been considered by Fröhlich in [4], where the definition used is somewhat different although equivalent to that used here. The only varieties of d.g. near-rings we will use will be those satisfying additive laws.

We will be using presentations of groups in $\mathfrak{B}$, a variety of groups. Free groups possessing the universal property exist in varieties, $([8], \S 4)$. So we can speak of $\operatorname{Fr}(X)$, the free $\mathfrak{B}$ group on the set $X$. A presentation of a group $G \in \mathfrak{B}$ is a definition of $G$ given by

$$
G=G p\left\{X ; w_{i}\left(x_{1}^{(i)}, \cdots, x_{n(i)}^{(i)}\right)=0, i \in I\right\}
$$

which means that $G \cong \operatorname{Fr}(X) / K$ where $K$ is the normal closure of the set of words $\left\{w_{i}\left(x_{1}^{(i)}, \cdots, x_{n(i)}^{(i)} ; i \in I\right\}\right.$ in $\operatorname{Fr}(X)$. This corresponds to ordinary presentations, except that we omit from the relations $\left\{w_{i}\left(x_{1}^{(i)}, \cdots, x_{n(1)}^{(i)}\right) ; i \in I\right\}$ all the laws of $\mathfrak{B}$. We will also use the fact that free products of groups exist in $\mathfrak{B}$, the $\mathfrak{B}$ free product, generally called the verbal product ([8], definition 18.31, theorem 18.42).

We now prove a result which will enable us to link a variety of groups with a corresponding variety of d.g. near-rings. First we need

Lemma 1.1. Let $w\left(x_{1}, \cdots, x_{n}\right)$ be a word in $n$ variables. Then

$$
g w\left(r_{1}, \cdots, r_{n}\right)=w\left(g r_{1}, \cdots, g r_{n}\right)
$$

whenever $r_{1}, \cdots, r_{n}$ lie in the d.g. near-ring $(R, S), g \in G$ and $G$ is an $(R, S)$ group.

Proof. We prove this lemma by induction on the length $l\left(w\left(x_{1}, \cdots, x_{n}\right)\right)$ of the word $w\left(x_{1}, \cdots, x_{n}\right)$. If $l\left(w\left(x_{1}, \cdots, x_{n}\right)\right)=1$, then the result comes from the definition. So we assume that the lemma holds for all words with length at most $m$. Suppose $l\left(w\left(x_{1}, \cdots, x_{n}\right)\right)=m+1$. Then

$$
w\left(x_{1}, \cdots, x_{n}\right)=w^{\prime}\left(x_{1}, \cdots, x_{n}\right)+y_{j}
$$

where $y_{j}= \pm x_{j}$ and $l\left(w^{\prime}\left(x_{1}, \cdots, x_{n}\right)\right)=m$. Then

$$
\begin{aligned}
g w\left(r_{1}, \cdots, r_{n}\right) & =g\left(w^{\prime}\left(r_{1}, \cdots, r_{n}\right) \pm r_{j}\right) \\
& =w^{\prime}\left(g r_{1}, \cdots, g r_{n}\right) \pm g r_{j} \\
& =w\left(g r_{1}, \cdots, g r_{n}\right)
\end{aligned}
$$

using the induction hypothesis. This finishes the induction argument and the proof. 
Let $\mathfrak{B}$ be a variety of groups, and let $(R, S)$ be a d.g. near-ring. Then we define the variety $\mathfrak{B}$ of d.g. near-rings by $(R, S) \in \mathfrak{B}$ if $(R,+) \in \mathfrak{B}$. There will be no confusion in using the same symbol for a variety of groups and a variety of d.g. nearrings.

THEOREM 1.2. Let $(R, S)$ be a d.g. near-ring with a faithful representation on the $(R, S)$ group $G$. Let $G \in \mathfrak{B}$, a variety of groups. Then $(R, S) \in \mathfrak{B}$.

Proor. Let $\mathfrak{B}$ be defined by the set of words $\left\{w_{i}\left(x_{1}, \cdots, x_{n(i)}\right) ; i \in I\right\}$. If $r_{1}, \cdots, r_{n(i)} \in R$, then, by lemma 1.1 ,

$g w_{i}\left(r_{1}, \cdots, r_{n(i)}\right)=w_{i}\left(g r_{1}, \cdots, g r_{n(i)}\right)=0$ for all $g \in G$. As the representation of $(R, S)$ on $G$ is faithful, this shows that $w_{i}\left(r_{1}, \cdots, r_{n(i)}\right)=0$ for all choices of $r_{1}, \cdots, r_{n(t)}$ in $R$, all $i \in I$. Hence the set of words $\left\{w_{i}\left(x_{1}, \cdots, x_{n(i)}\right) ; i \in I\right\}$ is satisfied in $(R,+)$ for all $i \in I$ and so $(R, S) \in \mathfrak{B}$.

Theorem 1.2 provides the reason for considering groups in $\mathfrak{B}$ together with d.g. near-rings in $\mathfrak{B}$.

Before we start the work of constructing free $(R, S)$ groups, we will prove a resuit which reduces the amount of work needed to show that a given mapping between d.g. near-rings is a d.g. near-ring homomorphism.

THEOREM 1.3. Let $(R, S)$ and $(T, U)$ be two d.g. near-rings. If $\theta$ is a group homomorphism from $(T,+)$ into $(R,+)$ which is also a semigroup homomorphism from $U$ into $S$, then it is a d.g. near-ring homomorphism from $(T, U)$ into $(R, S)$.

Proof. Since $\theta$ is a group homomorphism from $(T,+)$ into $(R,+)$, we only need to show that $\theta$ is a semigroup homomorphism from $T$ into $R$. We first note that, if $u \in U$, then $(-u) \theta=-(u \theta)$. Now let $\varepsilon_{1} u_{1}+\cdots+\varepsilon_{n} u_{n}$ and $\eta_{1} v_{1}+\cdots+\eta_{m} v_{m}$ be two elements of $T$, where $u_{i}, v_{j} \in T$ and $\varepsilon_{t}= \pm 1=\eta_{j}$ for $1 \leqq i \leqq n, 1 \leqq j \leqq m$. Then

Also

$$
\begin{aligned}
& \left(\varepsilon_{1} u_{1}+\cdots+\varepsilon_{n} u_{n}\right)\left(\eta_{1} v_{1}+\cdots+\eta_{m} v_{m}\right) \\
& \quad=\left(\varepsilon_{1} u_{1}+\cdots+\varepsilon_{n} u_{n}\right) \eta_{1} v_{1}+\cdots+\left(\varepsilon_{1} u_{1}+\cdots+\varepsilon_{n} u_{n}\right) \eta_{m} v_{m} \\
& \quad=\eta_{1}\left(\varepsilon_{1} u_{1} v_{1}+\cdots+\varepsilon_{n} u_{n} v_{n}\right)+\cdots+\eta_{m}\left(\varepsilon_{1} u_{1} v_{m}+\cdots+\varepsilon_{n} u_{n} v_{m}\right) .
\end{aligned}
$$

$$
\begin{aligned}
& \left(\varepsilon_{1} u_{1}+\cdots+\varepsilon_{n} u_{n}\right) \theta\left(\eta_{1} v_{1}+\cdots+\eta_{m} v_{m}\right) \theta \\
& \quad=\left(\varepsilon_{1}\left(u_{1} \theta\right)+\cdots+\varepsilon_{n}\left(u_{n} \theta\right)\right)\left(\eta_{1}\left(v_{1} \theta\right)+\cdots+\eta_{m}\left(v_{m} \theta\right)\right)
\end{aligned}
$$

as $\theta$ is a group homomorphism,

$$
\begin{aligned}
& =\left(\varepsilon_{1}\left(u_{1} \theta\right)+\cdots+\varepsilon_{n}\left(u_{n} \theta\right)\right) \eta_{1}\left(v_{1} \theta\right)+\cdots+\left(\varepsilon_{1}\left(u_{1} \theta\right)+\cdots+\varepsilon_{n}\left(u_{n} \theta\right)\right) \eta_{m}\left(v_{m} \theta\right) \\
& =\eta_{1}\left(\varepsilon_{1}\left(u_{1} \theta\right)\left(v_{1} \theta\right)+\cdots+\varepsilon_{n}\left(u_{n} \theta\right)\left(v_{1} \theta\right)\right)+\cdots+\eta_{m}\left(\varepsilon_{1}\left(u_{1} \theta\right)\left(v_{m} \theta\right)+\cdots+\varepsilon_{n}\left(u_{n} \theta\right)\left(v_{m} \theta\right)\right) \\
& =\eta_{1}\left(\varepsilon_{1}\left(u_{1} v_{1}\right) \theta+\cdots+\varepsilon_{n}\left(u_{n} v_{1}\right) \theta\right)+\cdots+\eta_{m}\left(\varepsilon_{1}\left(u_{1} v_{m}\right) \theta+\cdots+\varepsilon_{n}\left(u_{n} v_{m}\right) \theta\right)
\end{aligned}
$$


as $\theta$ is a semigroup homomorphism from $U$ into $S$,

$=\eta_{1}\left(\varepsilon_{1}\left(u_{1} v_{1}\right)+\cdots+\varepsilon_{n}\left(u_{n} v_{1}\right)\right) \theta+\cdots+\eta_{m}\left(\varepsilon_{1}\left(u_{1} v_{m}\right)+\cdots+\varepsilon_{n}\left(u_{n} v_{m}\right)\right) \theta$

as $\theta$ is a group homomorphism,

$=\left(\left(\varepsilon_{1} u_{1}+\cdots+\varepsilon_{n} u_{n}\right) \eta_{1} v_{1}\right) \theta+\cdots+\left(\left(\varepsilon_{1} u_{1}+\cdots+\varepsilon_{n} u_{n}\right) \eta_{m} v_{m}\right) \theta$

$=\left(\left(\varepsilon_{1} u_{1}+\cdots+\varepsilon_{n} u_{n}\right)\left(\eta_{1} v_{1}+\cdots+\eta_{m} v_{m}\right)\right) \theta$.

We have used the fact that if $s \in S$, then $r(-s)=-(r s)$, and have shown that $\theta$ is a semigroup homomorphism from $T$ into $R$, hence completing the proof of the theorem.

\section{Free $(R, S)$ groups}

Throughout we will work within a given variety $\mathfrak{B}$, which we will consider both as a variety of groups and of d.g. near-rings as defined above.

Let $S$ be a multiplicative semigroup. We will first define the free $\mathfrak{B}$ d.g. nearring on the semigroup $S$. This has already been done for the variety of all groups by Fröhlich in [3]. We generalize his results to arbitrary varieties, and use a different method of proof. Let $X$ be a set. Define $\operatorname{Fr}(X, S)$ to be the free $\mathfrak{B}$ group on the set of symbols $\left\{x, s_{x} ; x \in X, s \in S\right\}$. For $t \in S$, we define $\bar{t}$ as an endomorphism of $\operatorname{Fr}(X, S)$ by

$$
x \rightarrow \cdot{ }_{x}, s_{x} \rightarrow(s t)_{x}
$$

for all $x \in X, s \in S$. As the symbols $\left\{x, s_{\star} ; x \in X, s \in S\right\}$ are a free generating set in $\mathfrak{B}$ for $\operatorname{Fr}(X, S)$, we can extend the map defined in (2.1) uniquely to be an endomorphism of $\operatorname{Fr}(X, S)$.

Let $S=\{\tilde{s} ; s \in S\}$. We will show that $S$ is a semigroup of endomorphisms of $\operatorname{Fr}(X, S)$, isomorphic as a multiplicative semigroup to $S$. To do this it is enough to show that $\overline{t_{1} t_{2}}=\overline{t_{1}} \overline{t_{2}}$ for all $t_{1} \in S, i=1,2$, and also that if $t_{1} \neq t_{2}$ then $\overline{t_{1}} \neq \overline{t_{2}}$. But

$$
\begin{gathered}
x \overline{t_{1} t_{2}}=\left(t_{1} t_{2}\right)_{x}=t_{1 x} \overline{t_{2}}=x \overline{t_{1}} \overline{t_{2}}, \\
s_{x} \overline{t_{1} t_{2}}=\left(s t_{1} t_{2}\right)_{x}=\left(s t_{1}\right)_{x} \overline{t_{2}}=s_{x} \overline{t_{1}} \overline{t_{2}}
\end{gathered}
$$

for all $x \in X, s \in S$. As this is a generating set for $\operatorname{Fr}(X, S),(2.2)$ shows that $\overline{t_{1} t_{2}}$ $=\overline{t_{1}} \overline{t_{2}}$. If $t_{1} \neq t_{2}$, then

$$
x \overline{t_{1}}=t_{1 x} \neq t_{2 x}=x \overline{t_{2}} .
$$

Hence $\overline{t_{1}} \neq \overline{t_{2}}$ and we have shown that $S$ is a semigroup of endomorphisms of $\operatorname{Fr}(X, S)$ isomorphic to $S$. Because of this we will omit - and write $s$ for the endomorphism of $\operatorname{Fr}(X, S)$ denoted by $s$ above. We now write $(\operatorname{Fr}(S), S)$ for the d.g. near-ring generated by the semigroup $S$ of endomorphisms of $\operatorname{Fr}(X, S)$. 
The following theorem generalizes Theorem 2.1 of [3].

THEOREM 2.1. (i) $\operatorname{Fr}(S)$ is a d.g. near-ring in $\mathfrak{B}$ generated by the distributive semigroup $S$;

(ii) $(\operatorname{Fr}(S),+)$ is the free $\mathfrak{B}$ group on the set $S$;

(iii) every $S$ group $H \in \mathfrak{B}$ is a $(\operatorname{Fr}(S), S)$ group;

(iv) every semigroup homomorphism $\theta$ of $S$ into $T$, where $(R, T)$ is a d.g. near-ring in $\mathfrak{B}$, can be extended to a d.g. near-ring homomorphism from $(\operatorname{Fr}(S), S)$ to $(R, T)$;

(v) $(F r(S), S)$ is uniquely determined to within d.g. near-ring isomorphism by (i) and either (ii) or (iii) or (iv).

Proof. (i) follows from the definition, and theorem 1.2.

(ii) Let $\varepsilon_{1} s_{1}+\cdots+\varepsilon_{n} s_{n}$ be a word in the elements of $S$, where $\varepsilon_{i}= \pm 1$, $1 \leqq i \leqq n$. Then

$$
x\left(\varepsilon_{1} s_{1}+\cdots+\varepsilon_{n} s_{n}\right)=\varepsilon_{1} s_{1 x}+\cdots+\varepsilon_{n} s_{n x} .
$$

As $S_{x}=\left\{s_{x} ; s \in S\right\}$ is part of a free $\mathfrak{B}$ basis of $\operatorname{Fr}(X, S)$, the right hand side of (2.3) is equal to 0 only if $\varepsilon_{1} y_{1}+\cdots+\varepsilon_{n} y_{n}$ is a law in $\mathfrak{B}$ ([8], Corollary 13.25). This suffices to prove (ii) since we already know by theorem 1.2 that $(\operatorname{Fr}(S),+) \in \mathfrak{B}$. In particular (2.3) shows that $G p\left\{S_{x}\right\} \cong(F r(S),+)$ for each $x \in X$, under the obvious isomorphism.

(iii) Let $H$ be an $S$ group. Then there is a semigroup homomorphism $\theta$ from $S$ into $\operatorname{End}(H)$. As $H \in \mathfrak{B}$, we have $(E(H),+) \in \mathfrak{B}$. As $S$ is a free $\mathfrak{B}$ basis of $(\operatorname{Fr}(S),+)$, we can extend the mapping $\theta: S \rightarrow \operatorname{End}(H)$ to be a group homomorphism from $(\operatorname{Fr}(S),+)$ into $(E(H),+)$. Then by theorem 1.3, $\theta$ is a d.g. nearring homomorphism from $(\operatorname{Fr}(S), S)$ to $(E(H), \operatorname{End}(H))$, i.e. $H$ is a $(\operatorname{Fr}(S), S)$ group.

(iv) As $(R, T)$ is in $\mathfrak{B}$, we have $(R,+) \in \mathfrak{B}$. Then the same argument as that used in (iii) gives the result.

(v) Let $(R, S)$ be any d.g. near-ring in $\mathfrak{B}$. By (iv) there is a d.g. near-ring epimorphism $\theta$ from $(F r(S), S)$ onto $(R, S)$ which extends the identity map on $S$. If $(R, S)$ satisfies (ii), then $(F r(S),+) \cong(R,+)$ under $\theta$ and so $(R, S) \cong(\operatorname{Fr}(S), S)$ as a d.g. near-ring. If $(R, S)$ satisfies (iii), then $\operatorname{Fr}(X, S)$ is an $(R, S)$ group and, by the argument used in (ii), $(R,+)$ is the free $\mathfrak{B}$ group on the set $S$. So $(R, S)$ satisfies (ii) and is d.g. near-ring isomorphic to $(\operatorname{Fr}(S), S)$. If $(R, S)$ satisfies (iv), it is immediate that $\theta$ has a two sided inverse which is a d.g. near-ring homomorphism, arising from the identity map from $S \subseteq(R, S)$ to $S \subseteq(\operatorname{Fr}(S), S)$. Hence $\theta$ is a d.g. near-ring isomorphism. This finishes the proof of the theorem.

We now consider an arbitrary d.g. near-ring $(R, S)$, generated by a distributive semigroup $S$. By theorem 2.1 (iv), the identity map on $S$ extends to a d.g. near-ring epimorphism $\theta$ from $(\operatorname{Fr}(S), S)$ to $(R, S)$. Let $\operatorname{Ker} \theta$, the kernel of this epimor- 
phism be the ideal $I$ of $(F r(S), S)$. Then $\overline{F r(X, S) I}$, the normal closure of $\operatorname{Fr}(X, S) I$ $=G p\{g r ; g \in F r(X, S), r \in I\}$ in $F r(X, S)$ is easily seen to be a normal $S$ subgroup, and hence an $(R, S)$ subgroup ([2], 1.2.3). This enables us to define

$$
\operatorname{Fr}(X, S) / \overline{\operatorname{Fr}(X, S) I}=\operatorname{Fr}(X, R, S)
$$

as an $(R, S)$ group, where the action of $S$ on $\operatorname{Fr}(X, R, S)$ is defined in the natural way from the action of $S$ on $\operatorname{Fr}(X, S)$, namely

$$
(g+\overline{\operatorname{Fr}(X, S) I)} s=g s+\overline{F r(X, S) I} .
$$

To check that this defines $\operatorname{Fr}(X, R, S)$ as an $(R, S)$ group is a routine matter, and was essentially done by Fröhlich in [3], result (2.2). In the same paper, theorems 3.4 and 5.1, and in [4], theorem 2.4, Fröhlich establishes the existence of the free $(R, S)$ sum of $(R, S)$ groups and of free $(R, S)$ groups in a category. We will state and prove the result in the setting of varieties, as it is a fairly short result and for completeness.

THEOREM 2.2. $F r(X, R, S)$ is the free $(R, S)$ group on the set $X$, in the variety $\mathfrak{B}$.

Proof. Let $H$ be an $(R, S)$ group, and let $\theta: X \rightarrow H$ map $X$ into $H$. Define $\phi: F r(X, S) \rightarrow H$ by

$$
x \phi=x \theta, s_{x} \phi=(x \theta) s
$$

and extend this map to a homomorphism from $\operatorname{Fr}(X, S)$ to $H$, possible since $\operatorname{Fr}(X, S)$ is freely generated by $\left\{x, s_{x} ; x \in X, s \in S\right\}$. Then $H$ is an $(\operatorname{Fr}(S), S)$ group by theorem 2.1 (iii), and from (2.4), $\phi$ commutes with the action of $S$ on a generating set of $\operatorname{Fr}(X, S)$, and hence on $\operatorname{Fr}(X, S)$. Hence $\phi$ is an $S$ homomorphism, and so a $(F r(S), S)$ homomorphism ([2], 2.1.1). Since $H I=0$,

$$
(g r) \phi=(g \phi) r=0
$$

for all $g \in F r(X, S), r \in I$. Hence $\operatorname{Fr}(X, S) I \subseteq \operatorname{Ker} \phi$ and so $\overline{F r(X, S) I} \subseteq \operatorname{Ker} \phi$. Thus $\phi$ induces an $S$ homomorphism $\mu: \operatorname{Fr}(X, R, S) \rightarrow H$ which will also be defined by (2.4). Again $\mu$ is an $(R, S)$ homomorphism by [2], 2.1.1. Since $\mu$ must agree with $\theta$ on $X$, and is an $(R, S)$ homomorphism the definition (2.4) is forced and $\mu$ is uniquely defined. Hence the result is true.

If $S$ and hence $R$ has an identity, and we impose the condition that all representations of $(R, S)$ are to be unitary, then using theorems 5.7 and 5.8 of [3], or directly, it is easy to see that the following results hold.

THEOREM 2.3. Let $S$ be a semigroup with identity. Then $F r_{1}(X, S)$, the free $\mathfrak{B}$ group on the symbols $\left\{s_{x} ; x \in X, s \in S\right\}$ is the free unitary $(F r(S), S)$ group on $X$.

THEOREM 2.4. Let $S$ be a semigroup with identity. Then $F_{1}(X, R, S)$ $=F r_{1}(X, S) / \overline{F r_{1}(X, S) I}$ is the free unitary $(R, S)$ group on $X$, where 
$(R, S) \cong(F r(S), S) / I$ and $\overline{F r_{1}(X, S) I}$ is the normal closure of $F r_{1}(X, S) I=G p\{g r$; $\left.g \in F r_{1}(X, S), r \in I\right\}$ in $F r_{1}(X, S)$.

\section{The counterexample}

Before we proceed to construct our counterexample, we will prove two elementary results which will be needed at a later stage.

LEMMA 3.1. Let $(R, S) \in \mathfrak{B}$ have a faithful representation. Then the representation of $(R, S)$ on the free $(R, S)$ group on one generator is faithful.

Proof. Let $G \in \mathfrak{B}$ give rise to the faithful representation for $(R, S)$ and let $H=F r(x, R, S)$ be the free $(R, S)$ group on one generator, $x$. Given $r \neq 0, r \in R$, we can choose $g \in G$ such that $g r \neq 0$. Map $x \rightarrow g$ and extend this mapping to an $(R, S)$ homomorphism $\theta$ from $H$ to $G$ by theorem 2.2. Then $x r=0$ would imply

$$
0=(x r) \theta=(x \theta) r=g r \neq 0,
$$

a contradiction. Hence $x r \neq 0$ and $H$ gives rise to a faithful representation for $(R, S)$.

LEMMA 3.2. Let $(R, S)$ be a d.g. near-ring, and let $X=\left\{x_{\lambda} ; \lambda \in \Lambda\right\}$ be a set of elements in $R$. Then the ideal $I$ of $(R, S)$ generated by $X$ is the normal subgroup of $(R,+)$ generated by

$$
R X S=\left\{r x_{\lambda} s, r x_{\lambda}, x_{\lambda} s, x_{\lambda} ; \lambda \in \Lambda, r \in R, s \in S\right\} .
$$

Proof. If $I$ is the normal subgroup generated by $R X S$, it is easy to check that $R I \subseteq I$ and $I S \subseteq I$, and so, by Fröhlich [1], result 1.3.2, $I$ is an ideal. Any ideal containing $X$ must contain $R X S$ and hence $I$. This finishes the proof.

We now come to the counterexample. Let $\mathfrak{B}$ be any non-abelian variety. Let $S$ be a four element semigroup $S=\{a, b, c, 0\}$ with all products equal to 0 . Let $(F r(S), S)$ be the free d.g. near-ring in $\mathfrak{B}$ on the semigroup $S$, with 0 taken as the additive 0 of $(\operatorname{Fr}(S),+)$. We define $(R, S)$ as the homomorphic image of $(F r(S), S)$ given by the ideal $I$ generated by the element $a+b+c$ of $\operatorname{Fr}(S)$. In this case $(\operatorname{Fr}(S), S)$ is the zero d.g. near-ring. By lemma 3.2, $I$ is the normal closure of the element $a+b+c$ in $(\operatorname{Fr}(S),+)$ since $R X S$ reduces to $a+b+c$. Hence $(R,+)$ is the free $\mathfrak{B}$ group on two generators, which we can take to be $a$ and $b$.

Now let $G=F r(x, S)$ be the free $(F r(S), S)$ group on one generator, $x$, and let $H=F r(x, R, S)$ be the free $(R, S)$ group on the element $x$. Then $H=G / \overline{G I}$, where $\overline{G I}$ is the normal closure of $G I$ in $G$. Then the free $(R, S)$ generator of $H$ is $\bar{x}=x+\overline{G I}$. From lemma 3.1, we know that if $(R, S)$ has a faithful representation, then $\bar{x} r \neq 0$ for $0 \neq r \in R$. And then $\bar{x} R$ is a subgroup of $H$ isomorphic to $(R,+)$ under the mapping $r \rightarrow \bar{x} r$. 
But GI contains $x(a+b+c)$ and $(2 x)(a+b+c)=2 x a+2 x b+2 x c$. Hence in $H$ we have

and

$$
-\bar{x} c=\bar{x} a+\bar{x} b
$$

$$
-(2 \bar{x} c)=2 \bar{x} a+2 \bar{x} b .
$$

Hence $\bar{x} a$ and $\bar{x} b$ commute. As $\bar{x} R$ is generated by $\bar{x} a$ and $\bar{x} b$, it follows that $\bar{x} R$ is abelian. But $(R,+)$ is the free $\mathfrak{B}$ group on two generators, and $\mathfrak{B}$ is a nonabelian variety. Hence $(R,+)$ is not abelian, since any relation which holds between free generators of a free $\mathfrak{B}$ group is a law in $\mathfrak{B}([8], 13.25)$. Hence $\bar{x} R$ is not isomorphic to $(R,+)$ and we have a contradiction if we assume that $(R, S)$ has a faithful representation.

THEOREM 3.3. In every non-abelian variety $\mathfrak{B}$, there exists a d.g. near-ring $(R, S)$ which does not have a faithful representation.

The only restriction on $\mathfrak{B}$ is that it is not an abelian variety. So if $\mathfrak{B}$ is a locally finite variety, i.e. a variety whose finitely generated groups are finite, then we have a finite d.g. near-ring $(R, S)$ which does not have a faithful representation. Compare this with the results in Malone [5] and Malone and Heatherly [7], that any finite near-ring with a two sided zero can be embedded in a near-ring generated by the inner automorphisms of a suitable group. This gives a faithful representation of any finite d.g. near-ring $(R, S)$ in the wider sense of an embedding of $R$ as a nearring in a near-ring generated by the endomorphisms of some group $G$. But the elements of $S$ no longer remain distributive in the larger near-ring. The question as to whether a d.g. near-ring can be embedded in a d.g. near-ring with identity by means of a near-ring monomorphism is still open.

To return to d.g. near-ring embeddings, the question now arises as to what conditions on a d.g. near-ring $(R, S)$ are necessary or sufficient for $(R, S)$ to have a faithful representation. Two obvious sufficient conditions are

THEOREM 3.4. (i) If $S$ has a left identity, then any d.g. near-ring $(R, S)$ on $S$ has a faithful representation.

(ii) If $(R,+)$ has $S$ as a set of free $\mathfrak{B}$ generators, then $(R, S)$ has a faithful representation.

Proof. (i) The left identity of $S$ is a left identity for $R$, hence $(R,+)$ gives rise to a faithful representation for $(R, S)$

(ii) This follows from theorem 2.1.

These two results give examples of two kinds of sufficient conditions we may have: a condition on the multiplicative structure of $S$ or a condition on the presentation of $(R,+)$ in terms of $S$ as a set of generators. There would seem to be a lot of work to do in this direction. 


\section{The upper and lower faithful d.g. near-rings for $(R, S)$}

Throughout this section, we will work within a given variety $\mathfrak{B}$.

Although a d.g. near-ring $(R, S)$ may not have a faithful representation, it is the homomorphic image of a d.g. near-ring with a faithful representation, namely $(\operatorname{Fr}(S), S)$, and has as homomorphic image a d.g. near-ring with a faithful representation, in fact the representation on any $(R, S)$ group, including the free $(R, S)$ group. This motivates the following work.

We first define a faithful d.g. near-ring $(R, S)$ to be a d.g. near-ring $(R, S)$ with a faithful representation. We can now define the upper and lower faithful d.g. near-rings for a d.g. near-ring $(R, S)$.

The upper faithful d.g. near-ring for $(R, S)$ is a faithful d.g. near-ring $(\bar{R}, S)$ such that $\bar{\theta}:(\bar{R}, S) \rightarrow(R, S)$ is a d.g. near-ring epimorphism with $\left.\bar{\theta}\right|_{S}=$ identity, and if $\theta$ is any d.g. near-ring epimorphism from a faithful d.g. near-ring $(T, S)$ to $(R, S)$ with $\left.\theta\right|_{S}=$ identity, then $\theta=\phi \bar{\theta}$ for a uniquely defined d.g. near-ring epimorphism $\phi:(T, S) \rightarrow(\bar{R}, S)$.

The lower faithful d.g. near-ring for $(R, S)$ is a faithful d.g. near-ring $(R, S)$ such that $\underline{\theta}:(R, S) \rightarrow(\underline{R}, S)$ is a d.g. near-ring epimorphism with $\left.\underline{\theta}\right|_{s}=$ identity, and if $\theta$ is any d.g. near-ring epimorphism from $(R, S)$ to a faithful d.g. near-ring $(T, S)$ with $\left.\theta\right|_{S}=$ identity, then $\theta=\underline{\theta} \phi$ for a uniquely defined d.g. near-ring epimorphism $\phi:(\underline{R}, S) \rightarrow(T, S)$.

We will show that for any given d.g. near-ring $(R, S)$, upper and lower faithful d.g. near-rings exist, and we will determine them in terms of $(R, S)$. We start with the lower faithful d.g. near-ring. First we need some preliminary results.

LEMMA 4.1. Let $\theta$ be a d.g. near-ring homomorphism from $(R, S)$ into $(T, U)$. Let $G$ be a $(T, U)$ group with representation $\phi$. Then $G$ can be defined as an $(R, S)$ group, and the kernel of the representation $\mu$ of $(R, S)$ on $G$ is the inverse image under $\theta$ of $\operatorname{Ker} \phi$.

Proof. We define $r \mu$ by $g(r \mu)=g(r \theta \phi)$ for all $r \in R$, i.e. $\mu=\theta \phi$. The rest follows easily.

Lemma 4.2. Let $(R, S)$ be a d.g. near-ring, and let $G=F r(x, R, S)$ be the free $(R, S)$ group on one element, $x$. Write $A=A(R, S)=\{r ; G r=0\}$. If $\theta$ is a representation of $(R, S)$, then $\operatorname{Ker} \theta \supseteq A$.

Proof. Assume that $\operatorname{Ker} \theta \neq A$. Let $r \in A-\operatorname{Ker} \theta$. Then we have a group $H$ such that $h r \neq 0$ for some $h \in H$. Map $x$ to $h$ and extend this to $\phi$, an $(R, S)$ homomorphism from $G$ to $H$. Then

$$
0=(x r) \phi=(x \phi) r=h r \neq 0 .
$$

This is a contradiction, and so we deduce that $\operatorname{Ker} \theta \supseteq A$. 
THEOREM 4.3. Let $(R, S)$ be a d.g. near-ring. Then its lower faithful d.g. near-ring is $(R, S) / A$ where $A=A(R, S)=\{r ; G r=0\}$ and $G$ is the free $(R, S)$ group on one generator.

Proof. Certainly $(R, S) / A$ is a faithful d.g. near-ring. Denote $(R, S) / A$ by $(\underline{R}, S)$ and let $\underline{\theta}$ be the natural d.g. near-ring epimorphism from $(R, S)$ to $(\underline{R}, S)$ with $\left.\underline{\theta}\right|_{S}$ $=$ identity. Let $\theta$ be a d.g. near-ring epimorphism from $(R, S)$ to a faithful d.g. near-ring $(T, S)$ with $\left.\theta\right|_{S}=$ identity. Let $H=F r(x, T, S)$ be the free $(T, S)$ group on one element $x$. Then as $(T, S)$ is a d.g. homomorphic image of $(R, S)$, we deduce by lemma 4.1 that $H$ is an $(R, S)$ group with the action of $s \in S$ defined in the same way for both $(R, S)$ and $(T, S)$. This gives rise to a representation of $(R, S)$ whose kernel is $\operatorname{Ker} \theta$, again by lemma 4.1. But by lemma 4.2, this means that $A \subseteq \operatorname{Ker} \theta$. Hence $\theta=\underline{\theta} \phi$ for a d.g. near-ring epimorphism $\phi:(\underline{R}, S) \rightarrow(T, S)$. As $\theta=\underline{\theta} \phi$, we must have $\left.\phi\right|_{s}=$ identity and this will define $\phi$ uniquely since $S$ generates $(\underline{R},+)$.

We now turn to the upper faithful d.g. near-ring. Again, we will first prove some preliminary results.

LeMma 4.4. Let $(T, S)$ be a faithful d.g. near-ring, and let $G=F r(x, T, S)$. Then $G=G_{1} * G_{2}$ is the free $\mathfrak{B}$ product of $G_{1}$, the free $\mathfrak{B}$ group on one generator and $G_{2}$, a group isomorphic to $(T,+)$.

Proof. Let $(F, S)=(F r(S), S)$ be the free $\mathfrak{B}$ d.g. near-ring on the semigroup $S$, and let $H$ be the free $(F, S)$ group on one element $x$. By theorem 2.1, $H$ is the free $\mathfrak{B}$ group on the set $\left\{x, s_{\lambda} ; s \in S\right\}$. Also $(T, S)=(F, S) / I$ for some ideal $I$ of $(F, S)$ and $G=H /(H I)^{H}$, where $(H I)^{H}$ is the normal subgroup of $H$ generated by $H I=G p\{h r ; h \in H, r \in I\}$, by theorem 2.2. We write $H=H_{1} * H_{2}$ where $H_{1}=G p\{x\}, H_{2}=G p\left\{s_{s} ; s \in S\right\}$ are both free $\mathfrak{B}$ groups on the elements mentioned, and * indicates the free $\mathfrak{B}$ product. By the definition of the action of $(F, S)$ on $H$, we know that $H F=H_{2}$. Hence $H I \subseteq H_{2}$ and by lemma 4.5, we obtain $G \cong H_{1} * H_{2} /(H I)^{H_{2}}$, where $(H I)^{H_{2}}$ is the normal subgroup of $H_{2}$ generated by HI. Again $G T=H_{2} /(H I)^{H_{2}}$, identifying $G$ and $H_{1} * H_{2} /(H I)^{H_{2}}$. In fact $x T$ $=H_{2} /(H I)^{H_{2}} \cong(T,+)$ from the first part of the proof of theorem 3.3. Hence $G \cong H_{1} *(T,+)$ which is the result we want.

We now prove lemma 4.5 . It is a fairly standard result, but there does not seem to be a reference for it.

Lemma 4.5. Let $G=H * K$, let $N$ be normal in $K$ and let $M=N^{G}$ be the normal closure of $N$ in $G$. Then $G / M \cong H * K / N$, All groups are in $\mathfrak{B}$ and $*$ indicates the free $\mathfrak{B}$ product.

Proof. Let $G^{\prime}=H * K / N$. Map $G \rightarrow G^{\prime}$ by $\theta$ extending $h \rightarrow h, k \rightarrow k+N$. Then $\operatorname{Ker} \theta \supseteq N$. Hence $\operatorname{Ker} \theta \supseteq M$, the normal closure of $N$. We know that $\theta$ is a homomorphism by the universal property of free $\mathfrak{B}$ products $([8], 18.42)$. 
Now consider $G / M$. As $K^{G} \cap H=0$ ([8], 18.36), we have $M \cap H=0$. So if $\phi: G \rightarrow G / M$ is the natural homomorphism, then $H \phi=H M / M \cong H / H \cap M$ $=H$. Hence $G / M=G p\{H, K M / M\}$ where we are substituting $H$ for $H M / M$. We now define $\mu: G^{\prime} \rightarrow G / M$ by $h \rightarrow h$ and $k+N \rightarrow k+M$. Then $\mu$ extends to a homomorphism from $G^{\prime}$ to $G / M$, by the universal property of free products ([8], 18.42). It is immediate that $\mu$ is onto $G / M$. Hence $\theta \mu$ is an epimorphism from $G$ onto $G / M$ and

$$
h \theta \mu=h \mu=h, k \theta \mu=(k+N) \mu=k+M, \text { for all } h \in H, k \in K .
$$

Hence $\theta \mu=\phi$. So $\operatorname{Ker} \phi \supseteq \operatorname{Ker} \theta$, i.e. $M \supseteq \operatorname{Ker} \theta$. This means that $M=\operatorname{Ker} \phi$ $=\operatorname{Ker} \theta$. As $\theta \mu=\phi$, this means that $\mu$ is $1-1$ and so is an isomorphism.

We now return to our given d.g. near-ring $(R, S)$. Let $G=G p\{x\} *(R,+)$ $=G p\{x\} * R_{x}$, where we write $R_{x}$ for the subgroup of $G$ isomorphic to $(R,+)$ and $G p\{x\}$ is the free $\mathfrak{B}$ group on one generator. Define $s \in S$ as an endomorphism of $G$ by extending the map

$$
x \rightarrow s_{x}, r_{x} \rightarrow(r s)_{x}
$$

to the whole of $G$. This is possible by the universal property of free $\mathfrak{B}$ products ([8], 18.42), since $r_{x} \rightarrow(r s)_{x}$ is an endomorphism of $R_{x}$. It is easy to check that this defines a homomorphism from $S$ to a semigroup of endomorphisms of $G$ which we will still denote by $S$. Then $S$ generates a d.g. near-ring $(\bar{R}, S)$ which is faithful, with $G$ as a faithfuı representation.

THEOREM 4.6. The d.g. near-ring $(\bar{R}, S)$ defined above is the upper faithful d.g. near-ring for $(R, S)$.

Proof. Let $\varepsilon_{1} s_{1}+\cdots+\varepsilon_{n} s_{n}$ be a word in the elements of $S$ which is 0 in $\bar{R}$, where $\varepsilon_{x}= \pm 1,1 \leqq i \leqq n$. Then

$$
\begin{aligned}
0=x\left(\varepsilon_{1} s_{1}+\cdots+\varepsilon_{n} s_{n}\right) & =\varepsilon_{1} s_{1 x}+\cdots+\varepsilon_{n} s_{n x} \\
& =\left(\varepsilon_{1} s_{1}+\cdots+\varepsilon_{n} s_{n}\right)_{x} .
\end{aligned}
$$

Hence $\varepsilon_{1} s_{1}+\cdots+\varepsilon_{n} s_{n}=0$ in $R$. So the identity map on $S$ extends to a homomorphism $\theta$ from $(\vec{R},+)$ to $(R,+)$ and it is a d.g. near-ring epimorphism by theorem 1.3.

Now let $\theta$ be a d.g. near-ring epimorphism from a faithful d.g. nearring $(T, S) \rightarrow(R, S)$ with $\left.\theta\right|_{S}=$ identity. Then $(T, S) / I \cong(R, S)$ for some ideal $I$ of $(T, S)$. By lemma 4.4, if $H=\operatorname{Fr}(x, T, S)$ is the free $(T, S)$ group on one generator, then $H \cong G p\{x\} * T_{x}$ with $T_{x} \cong(T,+)$. So $(R,+) \cong R_{x}$ is a homomorphic image of $T_{x}$, namely $R_{x} \cong T_{x} / I_{L^{*}}$. Then there is a homomorphism from $H$ to $G$ extending the map $x \rightarrow x, t_{x} \rightarrow t_{x} \mu$, where $\mu$ is the natural map from $T_{x}$ to $R_{r}$ induced by $\theta$. 
By the definition of the action of $S$ on $H$, we know that $I_{x}$ is an $S$ group, and hence so is $I_{x}^{H}$, its normal closure in $H$. Hence $H / I_{x}^{H}$ is a $(T, S)$ group and by lemma 4.5, $H / I_{x}^{H} \cong G p\{x\} * R_{x}=G$. The action of $S \subseteq T$ on $G$ is the same as the action of $S \subseteq \bar{R}$ on $G$. Hence $(\bar{R}, S)$ is a homomorphic image of $(T, S)$ under a d.g. nearring homomorphism $\phi$ which extends the identity map on $S$. Then $\theta=\phi \bar{\theta}$, and $\phi$ is uniquely defined as it must act as the identity map on $S$, a generating set for $(T,+)$. This finishes the proof.

We will close with a fairly straightforward, but interesting result about the adjoining of identities.

Lemma 4.7. Let $(R, S)$ be a faithful d.g. near-ring. Let $G=\operatorname{Fr}(x, R, S)$ be the free $(R, S)$ group on the generator $x$. If $(T, U)$ is the d.g. near-ring contained in $E(G)$, with $U=S \cup\{1\}$, where 1 is the identity map on $G$, then $(T,+) \cong G$.

Proof. As $(R, S) \subseteq(T, U)$ with $S \subseteq U,(T,+)$ gives rise to a faithful representation of $(R, S)$. So the map $x \rightarrow 1$ extends to an $(R, S)$ homomorphism $\theta$ from $G$ to $(T,+)$. Then $(x s) \theta=1 s=s$, 1.e. $s_{x} \theta=s$. So $G \theta \supseteq U$ and $\theta$ is an epimorphism. But $(T, U)$ has a faithful representation on $G$. Consider the map $\phi: r \rightarrow x r$. This is a homomorphism from $(T,+)$ to $G$ such that $1 \phi=x 1=x$ and $s \phi=x s$ $=s_{x}$. Hence $\theta \phi$ is the identity map on $\left\{x, s_{x} ; s \in S\right\}$ a generating set for $G$, and $\phi \theta$ is the identity map on $U$, a generating set for $(T,+)$. So $\theta$ and $\phi$ are both isomorphisms, giving us the result we want.

We now compare this with lemma 4.4 which states that $G=G_{1} * G_{2}$ with $G_{1}=G p\{x\}$ the free $\mathfrak{B}$ group on one generator, and $G_{2} \cong(R,+)$. From lemma 4.7, $G_{2}=G p\left\{S_{x}\right\} \cong(R,+)$ under the isomorphism $s_{x} \rightarrow s$. So $(T, U)$, the d.g. near-ring obtained in the natural way by adjoining an identity to $(R, S)$, where $(R, S)$ is a faithful d.g. near-ring can be characterized as follows: $(T,+)$ is the free $\mathfrak{B}$ product of $(R,+)$ with a free $\mathfrak{B}$ group on one generator, namely 1 , and multiplication is determined by $U=S \cup\{1\}$ where 1 acts as multiplicative identity. If we now let $\mathfrak{B}$ be the variety of abelian groups, then the free $\mathfrak{B}$ group on one generator is a copy of $Z$, the integers under addition, the free $\mathfrak{B}$ product is the direct sum and we can see that we have the standard method for adjoining an identity. Of course in an abelian variety, d.g. near-rings are rings, and all rings are faithful. So this process can always be carried out for rings.

\section{References}

[1] A. Fröhlich, 'Distributively generated near-rings (I): Ideal theory', Proc. London Math. Soc. (3) 8 (1958), 74-94.

[2] A. Fröhlich, 'Distributively generated near-rings (II): Representation theory', Proc. London Math. Soc. (3) 8 (1958), 95-108.

[3] A. Fröhlich, 'On groups over a d.g. near-ring (I): Sum constructions and free R-groups', Quart.J. Math. (Oxford) (2) 11 (1960), 193-210. 
[4] A. Fröhlich, 'On groups over a d.g. near-ring (II): Categories and Functors', Quart. J. Math. (Oxford) (2) 11 (1960), 211-228.

[5] J. J. Malone, 'A near ring analogue of a ring embedding theorem', J. Algebra 16 (1970), 237-238.

[6] J. J. Malone and H. E. Heatherly, 'Some near-ring embeddings', Quart. J. Math. (Oxford) (2) 20 (1969), 81-85.

[7] J. J. Malone and H. E. Heatherly, 'Some near-ring embeddings (II)', Quart. J. Math. (Oxford) (2) 21 (1970), 445-448.

[8] Hanna Neumann, Varieties of Groups (Springer, Berlin, 1967).

Mathematical Institute

University of Edinburgh

Edinburgh, Scotland. 